\title{
Effects of NOB1 on the pathogenesis of osteosarcoma and its expression on the chemosensitivity to cisplatin
}

\author{
LEIMING LUO, YUANHUI WANG, YIRAN YIN, JIANHUA GE and XIAOBO LU \\ Department of Bone and Joint Surgery, The Affiliated Hospital of \\ Southwest Medical University, Luzhou, Sichuan 646000, P.R. China
}

Received July 25, 2017; Accepted November 23, 2017

DOI: $10.3892 / \mathrm{ol} .2018 .7730$

\begin{abstract}
The effects of NIN1/RPN12 binding protein 1 homolog (NOB1) on the pathogenesis of osteosarcoma and its expression on the chemosensitivity to cisplatin were investigated. Seventy-four patients with osteosarcoma who received surgical resection in The Affiliated Hospital of Southwest Medical University (Sichuan, China) from September 2013 to September 2016 were enrolled in this study. The expression of NOB1 in cancer and cancer-adjacent tissues of patients was detected by reverse transcription-polymerase chain reaction, and the relationship between NOB1 expression and the pathogenesis of osteosarcoma was analyzed. The expression of NOB1 in osteosarcoma MG-63 cells was interfered with using small interfering ribonucleic acid (siRNA). Western blotting was used to detect the transfection efficiency and changes in apoptosis indicators. Cell Counting Kit-8 (CCK-8) assay was used to examine changes in the sensitivity of cells to cisplatin. The effect of NOB1 knockout on cell apoptosis was examined by flow cytometry. In patients with osteosarcoma, the level of NOB1 mRNA in cancer tissues was significantly higher than that in cancer-adjacent tissues $(\mathrm{p}<0.05)$, and the expression of NOB1 was correlated with Ennecking staging and tumor size $(\mathrm{p}<0.05)$. The expression level of the apoptotic indicator caspase-3 was activated after siRNA interfered with NOB1 expression, thus reducing the expression level of anti-apoptotic indicator B-cell lymphoma 2. CCK-8 results showed that the downregulation of NOB1 increased the sensitivity of MG-63 cells to cisplatin $(\mathrm{p}<0.05)$. In addition, flow cytometry showed that the downregulation of NOB1 significantly promoted the apoptosis of MG-63 cells. NOB1 is significantly upregulated in patients with osteosarcoma, thus reducing the curative effect of cisplatin chemotherapy, which indicates that the prognosis is poor.
\end{abstract}

Correspondence to: Professor Xiaobo Lu, Department of Bone and Joint Surgery, The Affiliated Hospital of Southwest Medical University, 25 Taiping Street, Luzhou, Sichuan 646000, P.R. China E-mail: luxb0366@163.com

Key words: NOB1, osteosarcoma, cisplatin

\section{Introduction}

Human NIN1/RPN12 binding protein 1 homolog (NOB1) gene is located on chromosome $16 \mathrm{q} 22.1$ and expresses the $50 \mathrm{kDa}$ protein NOB1. NOB1 is frequently expressed in the liver, lung, and spleen and mainly located in the nucleus $(1,2)$. It was reported that NOB1 is abnormally expressed in invasive ductal carcinoma and may be involved in the occurrence and development of tumors (3). The expressions of NOB1 messenger RNA (mRNA) and protein in papillary thyroid carcinoma are significantly higher than those in normal thyroid tissues (4). In non-small cell lung cancer (NSCLC) and prostate cancer, NOB1 expression is also significantly correlated with tumornode-metastasis (TNM) staging, lymph node metastasis and histological grading $(5,6)$. However, the correlation between NOB1 expression in osteosarcoma and clinicopathological features of patients has not been reported.

In this study, in order to determine the potential role of NOB1 in osteosarcoma, reverse transcription-polymerase chain reaction (RT-PCR) was used to examine the expression of NOB1 in cancer and cancer-adjacent tissues of patients with osteosarcoma, and the correlation between NOB1 and prognosis was analyzed. Afterwards, the sensitivity of NOB1 to cisplatin was examined by the knockout of NOB1 expression in osteosarcoma cells using small interfering ribonucleic acid (siRNA), and the potential mechanism was analyzed.

\section{Patients and methods}

Clinical data. A total of 74 cases of paired osteosarcoma cancer and cancer-adjacent tissues were from specimens obtained by surgical resection from September 2014 to September 2016 in The Affiliated Hospital of Southwest Medical University (Sichuan, China) and all specimens were confirmed cases diagnosed by pathologists. Cancer-adjacent tissues refer to tissues more than $5 \mathrm{~cm}$ away from the tumor edge. None of the study patients received radiotherapy, chemotherapy or other treatment before operation. The mean age of the patients was $45 \pm 12.3$ years. According to Ennecking staging, they were divided into stage I $(n=28)$, stage II $(n=30)$ and stage III $(n=16)$. Tumors were located in the tibia $(n=41)$, the femur $(n=23)$ and other parts $(n=10)$. All the participating patients signed the informed consent form. The study was approved by the Ethics Committee of The Affiliated Hospital of Southwest Medical University. 
Detection of the expression of NOB1 in osteosarcoma by $R T$-PCR. Total RNA was isolated using TRIzol reagent (Invitrogen Life Technologies, Carlsbad, CA, USA) and reverse transcription was performed using a PrimeScript ${ }^{\circledR}$ RT reagent kit (Takara Biotechnology Co., Ltd., Dalian, China). RT-PCR was performed using a SYBR ${ }^{\circledR}$ Premix Ex Taq ${ }^{\mathrm{TM}}$ II kit (Takara Biotechnology Co., Ltd.) for 40 amplification cycles at $55^{\circ} \mathrm{C}$ annealing temperature. The relative quantification of each gene was analyzed by $2^{-\Delta \Delta C t}$ with glyceraldehyde 3-phosphate dehydrogenase (GAPDH) as internal reference for correction. The formula of the relative expression level of mRNA of each indicator is $2^{-\Delta \mathrm{Ct}}[\Delta \mathrm{Ct}=\mathrm{Ct}$ (target gene) $-\mathrm{Ct}$ (GAPDH)]. NOB1 and GAPDH primers were synthesized by Sangon Biotech Co., Ltd. (Shanghai, China). Primer sequences are as follows: NOB1 foward, 5-ATCTGCCCTACAAG CCTAAAC-3' and reverse, 5'-TCCTCCTCСТCСТCСТCAC-3; GAPDH forward, 5'-TGACTTCAACAGCGACACCCA-3' and reverse, 5'-CACCCTGTTGCTGTAGCCAAA-3'.

Cell culture. Human osteosarcoma cell line MG-63 was purchased from the American Type Culture Collection (ATCC; Manassas, VA, USA) and cultured in Dulbecco's modified Eagle's medium (DMEM) containing high-level sugar and $10 \%$ fetal bovine serum (FBS), then $100 \mu \mathrm{g} / \mathrm{ml}$ streptomycin and $100 \mathrm{IU} / \mathrm{ml}$ penicillin were added. The cell culture flask was placed in an incubator at $37^{\circ} \mathrm{C}$ containing $5 \% \mathrm{CO}_{2}$ with the humidity of $95 \%$.

siRNA interference. siRNA interference sequences of target NOB1 are listed in literature (7) and synthesized by GenePharma (Shanghai, China). Transfection was performed using siRNA transfection kit (Guangzhou RiboBio Co., Ltd., Guangzhou, China). Western blotting was used to validate silencing efficiency and select the optimal sequence of action. Three siRNA and si-control sequences are shown below.

Western blotting. Cells were lysed on ice for $1 \mathrm{~h}$ using radioimmunoprecipitation assay (RIPA) lysis buffer (Beyotime Institute of Biotechnology, Guangzhou, China), and the protein supernatant was extracted by centrifuging the cells for $1 \mathrm{~h}$ at 13,000 $\mathrm{x} \mathrm{g}$ for $30 \mathrm{~min}$. The protein concentration was measured with the bicinchoninic acid assay (BCA) protein concentration kit, and an appropriate amount of loading buffers (both from Beyotime Institute of Biotechnology), were added at $100^{\circ} \mathrm{C}$ for $5 \mathrm{~min}$. Each sample was electrophoresed at an equivalent amount of $40 \mu \mathrm{g}$. The protein was then transferred to a polyvinylidene fluoride (PVDF) membrane. Using 5\% skimmed milk at room temperature for $1 \mathrm{~h}$, rabbit anti-human NOB1 polyclonal was incubated by the main antibodies (dilution, 1:1,000; cat. no. 10091-2-AP; Proteintech Group, Inc., Chicago, IL, USA), rabbit antihuman caspase-3 and B-cell lymphoma 2 (Bcl-2) polyclonal antibodies (dilution, 1:1,000; cat. nos. ab13847 and ab59348; Abcam, Cambridge, UK). After membranes were washed with Tris-buffered saline with Tween-20 (TBST), NOB1 was incubated by the corresponding goat anti-rabbit horseradish peroxidase-labeled secondary polyclonal antibody (dilution, 1:5,000; cat. no. A0239; Beyotime Institute of Biotechnology). The membrane was visualized by the enhanced chemiluminescence (ECL) detection system (Bio-Rad Laboratories,

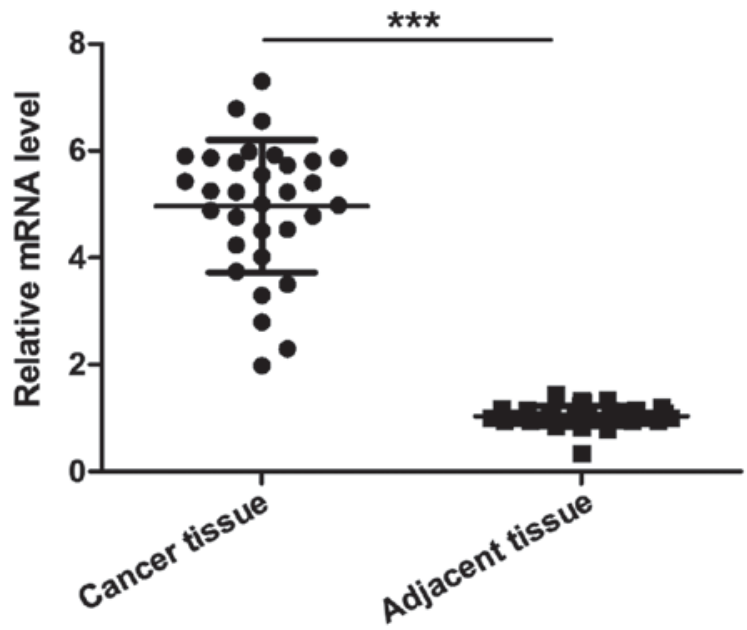

Figure 1. The level of NOB1 mRNA in cancer and cancer-adjacent tissues of patients with osteosarcoma. Cancer tissues vs. cancer-adjacent tissues; ${ }^{* * * *} \mathrm{p}<0.001$. NOB1, NIN1/RPN12 binding protein 1 homolog.

Inc., Hercules, CA, USA), and grayscale analysis was performed using a gel analyzer. The relative concentration of the target protein was the ratio of the target protein to the corresponding intrinsic parameter.

Detection of the sensitivity of MG-63 cells to cisplatin by Cell Counting Kit-8 (CCK-8). Cells were seeded in 96-well plates at $5 \times 10^{3} /$ well, and the cells were completely adherent $12 \mathrm{~h}$ later. The complete medium in each well was discarded. Two hundred microliters of prepared cisplatin at different concentrations $(0.001,0.01,1,10,20$ and $50 \mu \mathrm{M})$ were added after the culture for $24 \mathrm{~h}$. CCK-8 (20 $\mu \mathrm{l}$; Dojindo Molecular Technologies, Inc., Kumamoto, Japan) reagents were added to each well and incubated for $1 \mathrm{~h}$ at $37^{\circ} \mathrm{C}$ in the dark. The optical density (OD) at $450 \mathrm{~nm}$ was measured by a microplate reader. Cell viability $=(O D$ value of the experimental group - OD value of the blank group)/(OD value of the control group - OD value of the blank group) $\mathrm{x} 100 \%$.

Detection of apoptosis by flow cytometry. In this study, the detection was performed using cell apoptosis kits (BD Biosciences, San Jose, CA, USA) for testing. Cells were digested and centrifuged 2 days after transfection with siRNA, and then washed twice with cold phosphate-buffered saline (PBS). Afterwards, they were resuspended in $100 \mu \mathrm{l}$ of $1 \mathrm{X}$ binding buffer, and after $5 \mu \mathrm{l}$ propidium iodide (PI) and Annexin $\mathrm{V}$ were added, respectively, they were incubated at room temperature for $15 \mathrm{~min}$. Then, the cells were sent to the Scientific Research Center of our hospital for detection by apparatus within $1 \mathrm{~h}$. Apoptosis rate = early apoptosis rate + late apoptosis rate.

Statistical analysis. The results were analyzed by GraphPad Prism software (version 5.01; GraphPad Software Inc., La Jolla, CA, USA). Differences between two groups were compared by Student's t-test. Intergroup comparison of indicators was conducted using one-way analysis of variance (ANOVA). $\mathrm{P}<0.05$ was considered to indicate a statistically significant difference. 

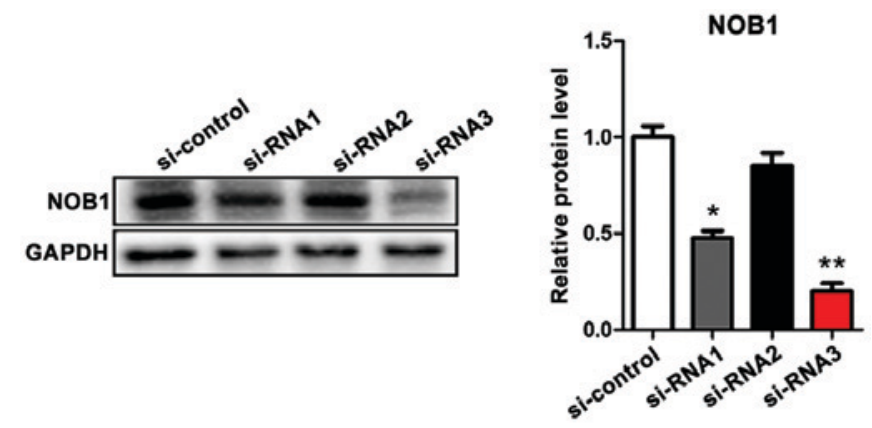

Figure 2. siRNA3 has the highest efficiency in target interference with NOB1. siRNA vs. si-control groups; ${ }^{*} \mathrm{p}<0.05,{ }^{* *} \mathrm{p}<0.01$. The downregulation of NOB1 by siRNA2 is not statistically significant ( $>0.05)$, and the effect of knocking out NOB1 is the most significant ( $\mathrm{p}<0.01)$. NOB1, NIN1/RPN12 binding protein 1 homolog.
A

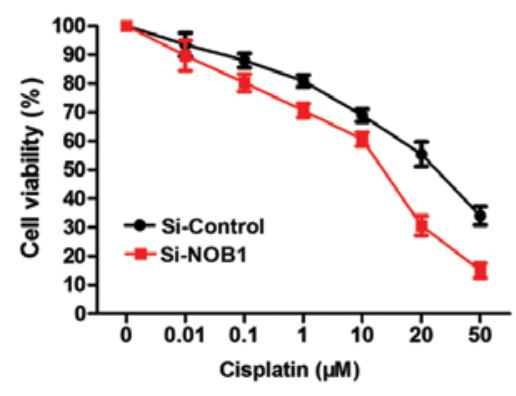

B

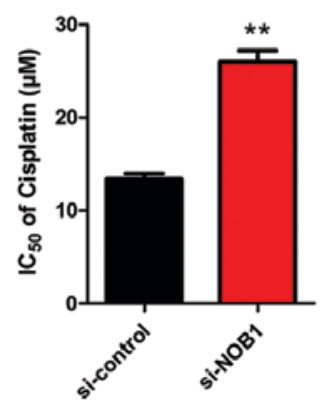

Figure 3. Detection of effects of NOB1 expression and osteosarcoma cells on the sensitivity to cisplatin by CCK-8. IC $_{50}$ : si-NOB1 vs. si-control $=12.23 \pm 2.74$ vs. $26.67 \pm 3.86 ;{ }^{* *} \mathrm{p}<0.01 . \mathrm{IC}_{50}$ : si-NOB1 vs. si-control, $12.23 \pm 2.74$ vs. $26.67 \pm 3.86 ;{ }^{* *} \mathrm{p}<0.01$. NOB1, NIN1/RPN12 binding protein 1 homolog; $\mathrm{IC}_{50}$, inhibitory concentration.
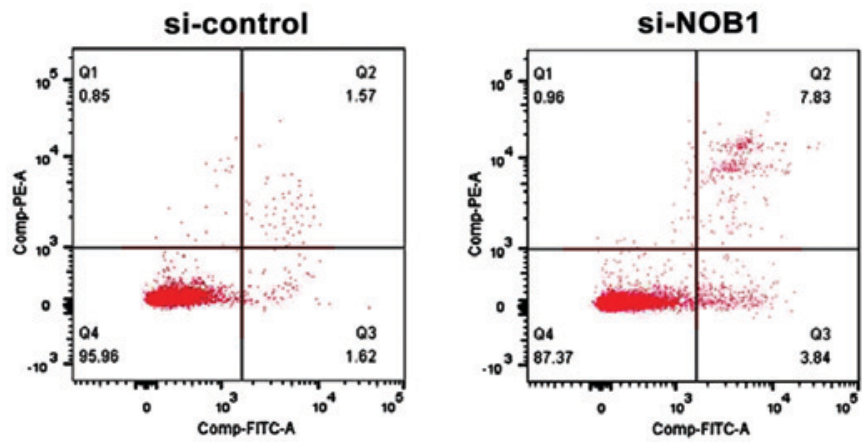

Figure 4. Detection of the correlation between NOB1 expression and osteosarcoma cell apoptosis by flow cytometry. Apoptosis rate: si-NOB1 vs. si-control: $12.54 \pm 1.58$ vs. $4.24 \pm 1.03 ; \mathrm{p}<0.05$. NOB1, NIN1/RPN12 binding protein 1 homolog.

\section{Results}

Detection of the expression of NOB1 mRNA in cancer and cancer-adjacent tissues of patients with osteosarcoma by $R T-P C R$. The level of NOB1 mRNA in cancer tissues was significantly higher than that in cancer-adjacent tissues of patients with osteosarcoma, and the difference was statistically significant $(\mathrm{p}<0.001)$.
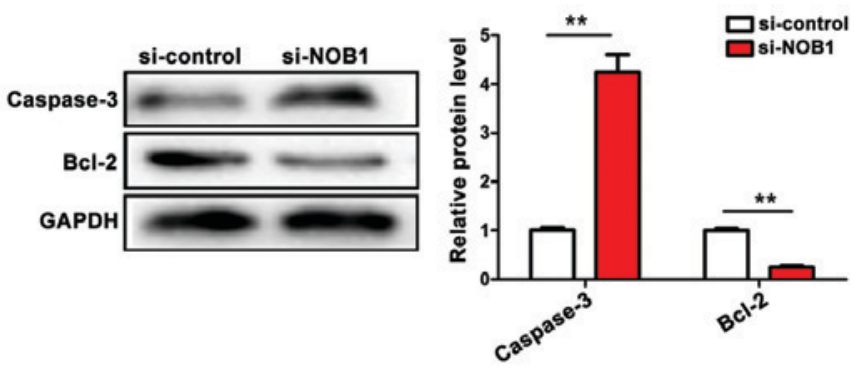

Figure 5. Detection of the effect of NOB1 expression on cell apoptosis by western blotting. si-NOB1 vs. si-control; ** $p<0.01$. NOB1, NIN1/RPN12 binding protein 1 homolog.

Correlation between the expression of NOB1 and the prognosis of patients with osteosarcoma (mean $\pm S D$ ). As shown in Fig. 1, the expression of NOB1 was not related to the age, sex, tumor location and lung metastasis $(\mathrm{p}>0.05)$ but correlated with Ennecking staging and tumor size, and the difference was statistically significant $(\mathrm{p}<0.05)$, suggesting that NOB1 was associated with the condition of patients with osteosarcoma.

Verification of the efficiency of three siRNAs in interfering with the expression of NOB1 in osteosarcoma cells (MG-63) by western blotting. Clinical experiments suggested that NOB1 mRNA was upregulated in cancer tissues of patients with osteosarcoma and was significantly associated with poor prognosis. In order to further study the chemosensitivity of NOB1 and osteosarcoma to cisplatin, three siRNAs were constructed for target interference with NOB1 expression in MG-63 cells. Western blotting results showed that siRNA2 had the highest efficiency in downregulating NOB1, and in the follow-up study, siRNA3 was studied (Fig. 2).

Detection of the sensitivity of MG-63 cells to cisplatin by $C C K-8$. As shown in Fig. 3, the sensitivity of MG-63 cells in the NOB1 downregulation group was significantly higher than that in the control group $(\mathrm{p}<0.01)$, suggesting that the expression of NOB1 in osteosarcoma cells was negatively correlated with the sensitivity to cisplatin.

Detection of the effect of NOB1 downregulation on apoptosis of MG-63 cells by flow cytometry. Annexin V and PI staining were used to mark early and late apoptosis cells. The results showed that NOB1 knockdown significantly increased the apoptosis rate of osteosarcoma MG-63 cells: si-NOB1 vs. si-control $=12.54 \pm 1.58$ vs. $4.24 \pm 1.03(\mathrm{p}<0.05)$, suggesting that NOB1 was closely related to the apoptosis level of osteosarcoma cells (Fig. 4).

Detection of the effect of NOB1 downregulation on apoptosis indicators of MG-63 cells by western blotting. The downregulation of NOB1 expression in osteosarcoma MG-63 cells activated the apoptosis pathway of caspase-3 [(si-NOB1 vs. si-control $=4.32 \pm 0.56$ vs. $1.02 \pm 0.03(\mathrm{p}<0.01)]$ and inhibited the expression of anti-apoptotic indicator Bcl-2 [(si-NOB1 vs. si-control $=0.22 \pm 0.02$ vs. $1.03 \pm 0.02(\mathrm{p}<0.01)$ ]. The downregulation of NOB1 expression promoted apoptosis of MG-63 cells, and the difference was statistically significant (Fig. 5). $(\mathrm{p}<0.05)$. 


\section{Discussion}

Osteosarcoma originates from mesenchymal cells formed by original bones, and is the most common primary bone malignancy (8). It mostly occurs in adolescents under the age of 20 years and children. In children and adolescents, $8.9 \%$ of deaths are caused by bone and joint malignancies. Studies have shown that the long-term survival rate of patients who only underwent surgical resection of osteosarcoma was $<20 \%$ (9). At present, cisplatin-based multidrug chemotherapy has greatly improved the prognosis of patients, and the 5-year survival rate with non-metastasis is $60-70 \%$ (10). However, chemotherapy resistance is still a problem in clinical treatment of osteosarcoma.

In normal cells, the $20 \mathrm{~S}$ and $26 \mathrm{~S}$ proteasomes are important constituents of the proteolytic system of cytosolic and nuclear proteasomes (11). NOB1 has been proven to be involved in two key cellular biological processes. First, NOB1 accomplishes degradation of $20 \mathrm{~S}$ protease by regulating the biosynthesis of the $26 \mathrm{~S}$ proteasome, which means that NOB1 participates in ubiquitin-regulated protein degradation processes $(12,13)$. Second, at the nuclear outlet, NOB1 and 40S ribosome activate the 20S ribosomal ribonucleic acid (rRNA) precursor at the D site so as to promote its formation of mature $18 \mathrm{~S}$ rRNA (13-15), indicating that NOB1 participates in the protein synthesis process. The two functions show that NOB1 plays an important role in the balance of proteins. Abnormal expression of NOB1 in cells may lead to dysfunction in protein synthesis and protein degradation. Rapidly growing cancer cells require more proteins for deoxyribonucleic acid (DNA) replication and cell division, so NOB1 are often abnormally activated and plays a biological role in a variety of tumor cells.

Apoptosis is an important event that affects cell growth, and it is also an important way to kill tumor cells by radiotherapy and chemotherapy. Liu et al and Meng et al reported that silencing NOB1 enhances the sensitivity of thyroid tumor cells to radiotherapy and the anticancer activity of chemotherapeutic agents $(16,17)$. Recent studies have shown that the downregulation of NOB1 induces apoptosis in colon and lung cancer $(18,19)$. Bcl-2/Bax opens permeability transition pore of mitochondria and releases cytochrome $c$ to activate caspase-9, thus inducing apoptosis. In this study, it was found that NOB1 expression level in cancer tissues was higher than that in cancer-adjacent tissues of patients with osteosarcoma, which is consistent with current studies and reports. siRNA knockout was used to silence NOB1, and it was found that apoptosis of MG-63 cells were significantly increased. Further mechanism studies have shown that NOB1 knockdown activates caspase- 3 expression and inhibits anti-apoptotic Bcl-2 expression. In addition, the sensitivity of MG-63 cells to cisplatin is also significantly enhanced, suggesting that NOB1 may be a potential target for the treatment of osteosarcoma.

\section{References}

1. Zhou GJ, Zhang Y, Wang J, Guo JH, Ni J, Zhong ZM, Wang LQ, Dang YJ, Dai JF and Yu L: Cloning and characterization of a novel human RNA binding protein gene PNO1. DNA Seq 15: 219-224, 2004

2. Zhang Y, Ni J, Zhou G, Yuan J, Ren W, Shan Y, Tang W, Yu L and Zhao S: Cloning, expression and characterization of the human NOB1 gene. Mol Biol Rep 32: 185-189, 2005.
3. Li XY, Luo QF, Li J, Wei CK, Kong XJ, Zhang JF and Fang L: Clinical significance of NOB1 expression in breast infiltrating ductal carcinoma. Int J Clin Exp Pathol 6: 2137-2144, 2013.

4. Lin S, Meng W, Zhang W, Liu J, Wang P, Xue S and Chen G: Expression of the NOB1 gene and its clinical significance in papillary thyroid carcinoma. J Int Med Res 41: 568-572, 2013.

5. Liu K, Gu MM, Chen HL and You QS: NOB1 in non-small-cell lung cancer: Expression profile and clinical significance. Pathol Oncol Res 20: 461-466, 2014.

6. Liu G, Shen D, Jiao L and Sun Y: Nin one binding protein expression as a prognostic marker in prostate carcinoma. Clin Transl Oncol 16: 843-847, 2014.

7. Gao X, Wang J, Bai W, Ji W and Wang L: NOB1 silencing inhibits the growth and metastasis of laryngeal cancer cells through the regulation of JNK signaling pathway. Oncol Rep 35: 3313-3320, 2016.

8. Mirabello L, Troisi RJ and Savage SA: Osteosarcoma incidence and survival rates from 1973 to 2004: Data from the surveillance, epidemiology, and end results program. Cancer 115: 1531-1543, 2009.

9. Friedman MA and Carter SK: The therapy of osteogenic sarcoma: Current status and thoughts for the future. J Surg Oncol 4: 482-510, 1972.

10. Kawahara M, Takahashi Y, Takazawa K, Tsuchiya H, Tomita K, Yokogawa K and Miyamoto K: Caffeine dose-dependently potentiates the antitumor effect of cisplatin on osteosarcomas. Anticancer Res 28 (3A): 1681-1685, 2008.

11. Reinheckel T, Ullrich O, Sitte N and Grune T: Differential impairment of $20 \mathrm{~S}$ and $26 \mathrm{~S}$ proteasome activities in human hematopoietic K562 cells during oxidative stress. Arch Biochem Biophys 38: 65-68, 2000.

12. Tone Y1 and Toh-E: A Noblp is required for biogenesis of the $26 \mathrm{~S}$ proteasome and degraded upon its maturation in Saccharomyces cerevisiae. Genes Dev 16: 3142-3157, 2002.

13. Lamanna AC and Karbstein K: Nob1 binds the single-stranded cleavage site D at the 3'-end of 18S rRNA with its PIN domain. Proc Natl Acad Sci USA 106: 14259-14264, 2009.

14. Fatica A, Oeffinger M, Dlakić M and Tollervey D: Noblp is required for cleavage of the $3^{\prime}$ end of $18 \mathrm{~S}$ rRNA. Mol Cell Biol 23: 1798-1807, 2003.

15. Fatica A, Tollervey D and Dlakić M: PIN domain of Noblp is required for D-site cleavage in $20 \mathrm{~S}$ pre-rRNA. RNA 10 : 1698-1701, 2004.

16. Liu J, Dong BF, Wang PS, Ren PY, Xue S, Zhang XN, Han Z and Chen G: Silencing NOB1 enhances doxorubicin antitumor activity of the papillary thyroid carcinoma in vitro and in vivo. Oncol Rep 33: 1551-1559, 2015.

17. Meng W, Wang PS, Liu J, Xue S, Wang GM, Meng XY and Chen G: Adenovirus-mediated siRNA targeting NOB1 inhibits tumor growth and enhances radiosensitivity of human papillary thyroid carcinoma in vitro and in vivo. Oncol Rep 32: 2411-2420, 2014.

18. He XW, Feng T, Yin QL, Jian YW and Liu T: NOB1 is essential for the survival of RKO colorectal cancer cells. World J Gastroenterol 21: 868-877, 2015.

19. Li Y, Ma C, Qian M, Wen Z, Jing H and Qian D: Downregulation of NOB1 suppresses the proliferation and tumor growth of non-small cell lung cancer in vitro and in vivo. Oncol Rep 31: 1271-1276, 2014

This work is licensed under a Creative Commons Attribution-NonCommercial-NoDerivatives 4.0 International (CC BY-NC-ND 4.0) License. 